\title{
Diseño de un dispositivo de asistencia ventilatoria temporal de lazo cerrado basado en bolsa válvula-mascarilla
}

\author{
Design of a closed-loop temporary ventilation assistance device based \\ on bag valve mask \\ Jorge A. Balsells ${ }^{1 *}$, Luis G. García², Iván Morales ${ }^{3,6}$, Víctor López $^{4}$, Víctor Carranza ${ }^{5}$, Saúl López $^{5}$, \\ Astrid C. Villatoro ${ }^{6}$, Luis A. García 7 ,Juan C. Castillo ${ }^{7}$, Hasel Nájera ${ }^{7}, H_{\text {Hector Cabrera }}^{8}$ \\ ${ }^{1}$ Laboratorio de Innovación FabLab-DIGI, Universidad San Carlos de Guatemala, ${ }^{2}$ International Centre for Theoretical \\ Physics, Italia, ${ }^{3}$ Escuela de Ciencias Físicas y Matemáticas, Universidad San Carlos de Guatemala; ${ }^{4}$ Instituto Nacional \\ de Electrificación, Guatemala, ${ }^{5}$ Escuela de Ingeniería Mecánica Eléctrica, Facultad de Ingeniería, y ${ }^{6}$ Facultad de \\ Arquitectura, y ${ }^{7}$ Facultad de Ciencias Médicas, Universidad de San Carlos de Guatemala, \\ ${ }^{8}$ Hospital General San Juan de Dios, Guatemala
}

*Autor al que se dirige la correspondencia: jbalsells@digi.usac.edu.gt

Recibido: 15 de julio 2020 / Revisión: 16 de octubre 2020 / Aceptado: 18 de noviembre 2020

Resumen

$\mathrm{D}$ esde inicios del 2020, el mundo se ha visto afectado por la COVID-19 causada por el SARS-CoV-2, que en agosto lo padecen más de 31 millones de pacientes, algunos de los cuales presentan el síndrome de distrés respiratorio, que requiere de ventilación mecánica. Por el alto número de contagios, la disponibilidad de ventiladores para el tratamiento es escasa. Se presenta la descripción de un prototipo de un dispositivo de asistencia ventilatoria temporal de lazo cerrado de bajo costo; el AR_CODEX, basado en una bolsa válvula-mascarilla (BVM), que contribuye al mantenimiento ventilatorio mínimo del paciente durante un tiempo corto en casos donde no hay disponibilidad de ventiladores mecánicos. Para esto, se diseñó y construyó un sistema mecánico ajustable que compresiona la bolsa de ventilación, el cual cuenta con sensores de flujo y presión. Además, se elaboró una interfaz gráfica para un adecuado monitoreo del paciente y un sistema de control para variables como volumen, presión máxima, frecuencia respiratoria y relación inspiración:espiración. Por otro lado, existe un problema de sensibilidad en el sensor de flujo debido a varios factores, como la variación del voltaje en los motores. Adicionalmente, la implementación de un lazo cerrado es importante para compensar variaciones aleatorias en el funcionamiento del dispositivo. Es necesario realizar pruebas en animales para evaluar el correcto funcionamiento de AR_CODEX en seres vivos.

Palabras claves: Asistencia respiratoria automatizada, bajo costo, COVID-19, código abierto

\begin{abstract}
$\mathrm{I}$

n early 2020, the world has been affected from Covid-19 caused by SARS-CoV-2. By August there were more 1 than 31 million patients, some of them suffering from respiratory distress that requires mechanical ventilation. Due to the rise of infection rates there is no ventilator availability for the treatment. In this work we describe a reduced cost closed loop temporal assisted ventilation device prototype, AR_CODEX. It is based on mask valve bag (BVM from its Spanish initials), contributing to the minimum ventilation maintenance for the patient during a short period of time when there is no mechanical ventilation availability. For this purpose an adjustable mechanical system was designed and built to pressurize the ventilation bag that is equipped with flux and pressure sensors. Additionally a graphical interface was developed to include adequate monitoring and controlling system for volume, maximum pressure, respiratory frequency and inhalation/exhalation rate. In addition there is a sensibility issue on the flux sensor due to engine voltage variation. A closed loop implementation is important to overcome aleatory variations during the device operation. It is needed to run AR_CODEX device performance test on animals to evaluate prior to use it directly on human patients.
\end{abstract}

Keywords: Automated respiratory support, low cost, COVID-19, open source 


\section{Introducción}

En diciembre del 2019 dio inicio el brote de la enfermedad coronavírica 2019 (COVID-19), que es causada por el coronavirus del síndrome respiratorio severo agudo (SARS-CoV-2), surgiendo en la ciudad de Wuhan, China (Li \& Ma, 2020). La COVID-19 fue declarada el 11 de marzo de 2020 como pandemia por la Organización Mundial de la Salud (WHO, por sus siglas en inglés) (Preckel et al., 2020). Según datos de la WHO presentados en su página web oficial, a la fecha del 24 de septiembre de 2020 a las 5:05 pm CEST, se contabilizan un total de 31,798,308 casos confirmados; de los cuales 973,653 han resultado en decesos. Los países que presentan la mayoría de casos son: Estados Unidos, con 6,828,785 casos; India, con 5,732,518 casos y Brasil con 4,591,604 (World Health Organization [WHO], 2020).

La COVID-19 afecta principalmente al sistema respiratorio y algunos de los pacientes desarrollan el síndrome de distrés respiratorio agudo (ARDS, acute respiratory distress syndrome), en el que el resto de sistemas se ven comprometidos en menor medida (Li \& Ma, 2020). Hay datos que indican que cerca del $25.9 \%$ de los pacientes con neumonía por SARS-CoV-2 requieren admisión a las unidades de cuidados intensivos y el 20.1\% desarrollan ARDS (Cunningham et al., 2020). La letalidad relacionada a COVID-19 es dependiente de la edad, se reportan tasas desde $<0.6 \%$ hasta $2.2 \%$ a la edad de 60 años, incrementándose hasta un $9.3 \%$ a edades de 80 años (Whittle et al., 2020).

El ARDS se define como la instauración aguda de infiltrado pulmonar bilateral, hipoxemia severa y edema pulmonar que no es explicado satisfactoriamente por causas cardíacas o por excesos de fluidos (Berlin et al., 2020). Según las directrices del manejo clínico del ARDS los pacientes con COVID-19 que desarrollan ARDS pueden verse beneficiados por la instauración de soporte ventilatorio mecánico invasivo. En líneas generales, se recomienda ajustar los parámetros ventilatorios con una estrategia protectora para los pulmones; con un volumen corriente $(\mathrm{Vt}) \mathrm{de}$

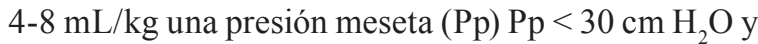
una presión positiva al final de la inspiración (PEEP) alta (L. Li et al., 2020).

En la situación actual derivada de la pandemia de COVID-19 se ha incrementado la cantidad de pacientes que requieren de cuidados respiratorios. Los sistemas de salud poseen un inventario limitado de ventiladores mecánicos y sus componentes. Una de las principales razones para la carencia de ventiladores es debido al rápido aumento en las necesidades de estos por la pandemia, en la que muchos de los países productores han detenido sus exportaciones, tanto de ventiladores mecánicos como de sus componentes para repuesto. Por lo tanto, existe la necesidad de escalar la producción de ventiladores por parte de los fabricantes existentes y de los nuevos en potencia. Los ventiladores fabricados a partir de tecnologías de impresión 3D y open-source, se deben de explorar al máximo para sobrellevar la crisis sanitaria global (Iyengar et al., 2020).

La ventilación mecánica es un tratamiento de soporte vital en la cual se utiliza un dispositivo que genera un diferencial de presión, lo cual genera un flujo de aire durante un tiempo determinado. El flujo depende de la relación entre las propiedades elásticas del sistema (elastancia del sistema respiratorio) y la presión generada por el dispositivo (Gutiérrez, 2011). Los ventiladores mecánicos son clasificados de acuerdo al parámetro que determina el fin del flujo inspiratorio, se clasifican como: (1) ventiladores ciclados por presión, que finalizan el flujo cuando se alcanza una presión predefinida en las vías aéreas; (2) ventiladores ciclados por volumen, donde se suministra un determinado volumen de aire al paciente, sin embargo, se define una presión máxima para evitar el daño a los pulmones; (3) ventiladores ciclados por tiempo, donde se configura la duración de la inspiración y la tasa de flujo; y, (4) los ventiladores ciclados por flujo, donde la inspiración se finaliza cuando la tasa de flujo cae por debajo de un valor específico (Pearce, 2020).

Un ventilador mecánico debe de contar con: (1) panel de control, donde se programan los diferentes parámetros de trabajo y las alarmas, además se despliegan los datos de los sensores; (2) sistema electrónico, que permite la conversión de las señales analógicas/ digitales, control de las funciones, memoria y vigilancia; (3) sistema neumático, que es el encargado de mezclar los gases y, de medir el flujo, la presión y los volúmenes; (4) sistema de suministro eléctrico, que provee la energía eléctrica necesaria para el funcionamiento del dispositivo; (5) sistema de suministro de gases; (6) circuito del paciente, que conecta al respirador mecánico con el paciente y está conformado por las mangueras corrugadas y los filtros; y, (7) sistema de alarmas, que idealmente debe de ser visible y audible y cuenta con alarmas programables y no programables (Gutiérrez, 2011).

En el contexto de la pandemia de COVID-19 han existido iniciativas del sector académico para elabo- 
Jorge A. Balsells ... et al.

rar diversos dispositivos de apoyo ventilatorio opensource basados en una bolsa de ventilación manual (BVM) para tratar de mitigar la escasez de estos dispositivos en los servicios de salud. Además, contribuyen a reducir la exposición del personal médico a los aerosoles generados por el paciente, al minimizar el uso de ventilación manual y proveer presión positiva continua después de la intubación endotraqueal (Australian and New Zealand Intensive Care Society [ANZICS], 2020). Algunos de los trabajos más importantes son: (1) E-Vent, que está siendo desarrollado por un grupo del Instituto Tecnológico de Massachussets (MIT) del curso de diseño de precisión, el cual puede obtener su aprobación pronto de parte de la Agencia de Alimentos y Drogas de los Estados Unidos (FDA) (Whitwam, 2020); (2) El Monolithic Power Systems (MPS), ventilador open-source, que se está trabajando en el MIT por un equipo de ingenieros, posee controles operacionales para $\mathrm{Vt}$, frecuencia respiratoria, medición y monitoreo de presión, además de contar con un sistema de alarmas (Monolithic Power Systems, 2020) $y$, (3) Vent4US, que es un ventilador desarrollado en colaboración entre las Universidades de Utah y Stanford (H. Li et al., 2020).

AR_CODEX es un desarrollo experimental. El objetivo general es elaborar un prototipo de asistencia ventilatoria temporal automática de bajo costo utilizando una BMV como base, que sea ciclado por volumen y limitado por presión. Para que sea funcional, este dispositivo debe ser de lazo cerrado y redundante, esto significa tener el control de las variables necesarias para mantener una señal estable en cada una de las variables medidas y tener acceso a mantener ciertos controles cuando algo se obstruye o daña, tal es el caso de los motores. De igual manera se debe construir un sistema mecánico ajustable que compresione la bolsa de ventilación, el cual cuenta con sensores de flujo y presión, además de elaborar una interfaz gráfica para un adecuado monitoreo del paciente y un sistema de control para variables como volumen, presión máxima, frecuencia respiratoria y relación inspiración:espiración. Por otro lado, se propone que todo el procesamiento debe utilizar una unidad de procesamiento de bajo costo, que presente características dinámicas, de fácil acceso, utilice interfaces y protocolos estándares, y que los datos del proyecto se desplieguen utilizando una pantalla TFT (Thin-film-transistor liquid-crystal display) de 16 bits, con pantalla táctil resistiva, de baja resolución y en un centro de control por medio de software cliente-servidor.

\section{Presentación del Caso}

\section{Sistema mecánico}

El dispositivo utiliza dos motores Stepper NEMA 17 , con un torque de $0.55 \mathrm{~N} . \mathrm{m}$, que hacen girar una barra roscada que convierte el movimiento de rotación, generado por los motores, a traslación. Los motores están posicionados sobre un perfil de aluminio 2020 tipo V, que se desplaza linealmente con rodillos sobre el mismo (Figura 1). Además, para generar la compresión de la BVM, que se encuentra en medio, se dejan dos grados de libertad.

Se emplearon barras roscadas con rosca ACME con husillos T8. Estas barras roscadas convierten el movimiento lineal a rotacional y se basa en un tornillo de potencia para ganar torque a costa de la pérdida de velocidad. No obstante, esto se soluciona implementando un segundo motor que presiona paralelamente la bomba de aire. Además, su implementación también sirve para continuar funcionando en caso de que se presente algún problema mecánico.

Varias piezas del dispositivo están impresas con ácido poliláctico (PLA), porque es un biopolímero termoplástico biodegradable, no tóxico y biocompatible. Se ha utilizado en implantaciones médicas, bioingeniería, dispositivos ortopédicos, entre otros. Además, es un antimicrobiano que puede inhibir el crecimiento de microorganismos como bacterias, hongos o protozoos. Estas características aumentan la eficiencia y selectividad de los agentes antimicrobianos, al trabajar en conjunto con ellos para disminuir los peligros del ambiente (Pawar et al., 2014). Las piezas impresas en 3D han resistido el estrés mecánico generado por el dispositivo dado que tienen varias capas de paredes sólidas y una estructura interna hexagonal creada con una densidad de $35 \%$ de PLA y $65 \%$ de aire, lo cual otorga una mayor resistencia a cada una de las piezas sin necesidad de hacerlas muy pesadas.

El sistema mecánico consta de dos motores para poder duplicar la frecuencia respiratoria con la que operaría un solo motor y a la vez agregar redundancia en caso de fallas mecánicas, y de esta manera, mantener el dispositivo en un rango de operación seguro.

La estructura metálica está desarrollada en perfiles de aluminio tipo V(V-SLOT) y tipo T(T-SLOT) 2020, por la facilidad al momento de prototipar, los tornillos y tuercas son M3 y M4 de acero inoxidable. Estos materiales son necesarios para evitar óxido en cualquier parte del dispositivo. 


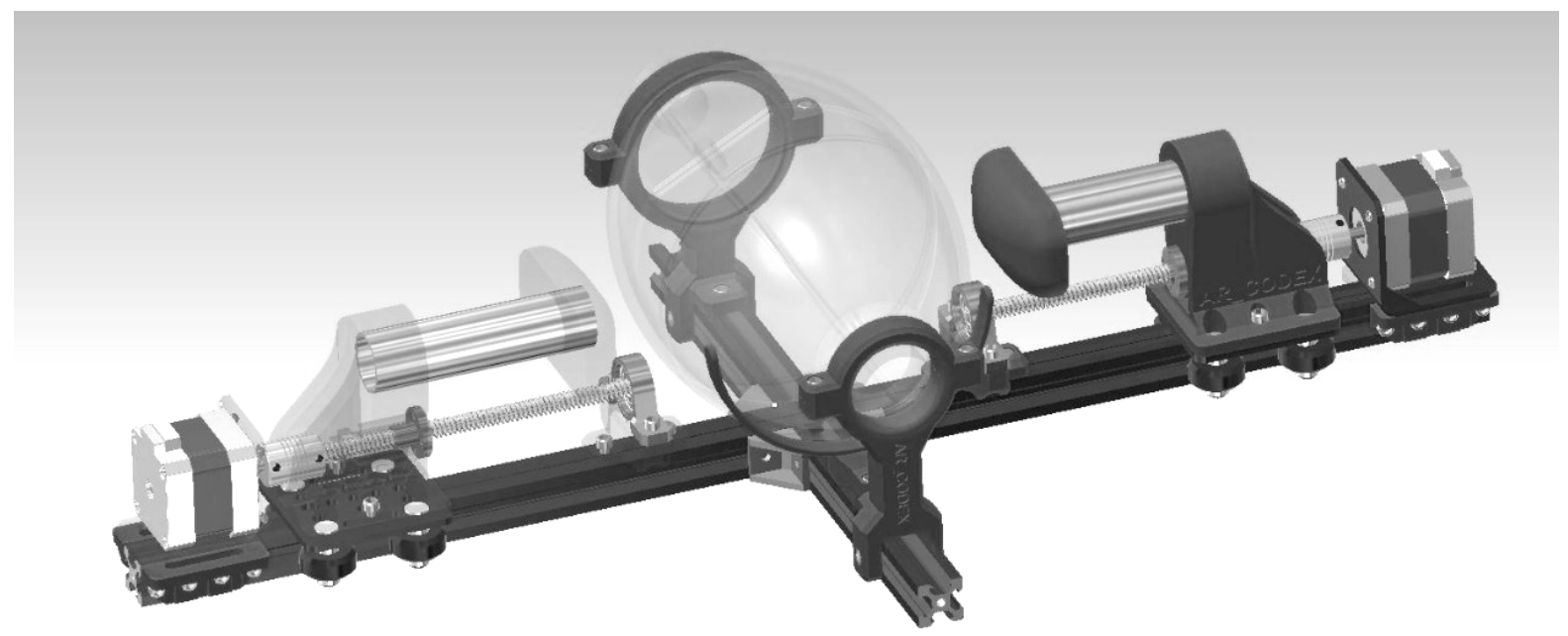

Figura 1. Estructura del desarrollo mecánico de AR_CODEX.

Por otro lado, se emplean tres microcontroladores, un Tensilica Xtensa LX6 (ESP32) y dos ATmega328P en un diseño modular, con el fin de distribuir la carga de procesamiento, evitando la interferencia de procesos, así como facilitar el intercambio de piezas para reparaciones o esterilización. El primer microcontrolador está encargado de la interfaz gráfica de los sensores que se colocan en el paciente y de enviar los datos a un servidor para su almacenamiento, alarmas y reportería. A su vez, el primer ATmega328P, se encarga de digitalizar las señales de los sensores de la BVM. El tercer microcontrolador es el encargado de recibir las señales de los sensores (previamente procesadas), mantener la ejecución de un sistema de control de lazo cerrado y controlar los actuadores. Se comunican entre sí utilizando un protocolo $\mathrm{I}^{2} \mathrm{C}$ (del inglés inter-integrated circuits). La medición de las señales del ventilador se basa en el principio de Bernoulli y el cálculo del flujo de aire se realiza por medio de la medición de la presión diferencial producida en un tubo de Venturi. Las señales de ambos sensores pasan por una fase de amplificación y corrección de offset, muestreadas por una de las entradas de conversión analógica a digital (ADC por sus siglas en inglés) de un microcontrolador Atmega328P.

\section{Sistema de sensores}

El diseño de AR_CODEX contempla la integración de sensores para monitorear al paciente. Entre los sensores podemos mencionar: temperatura, frecuencia cardiaca y saturación de oxígeno, integrados a un microcontrolador ESP32, el cual controla los parámetros de muestreo, la visualización y control utilizando una interfaz gráfica de usuario (GUI) en una pantalla táctil Thin Film Transistor (TFT) integrada al módulo, todos los datos son en tiempo real. El módulo integra comunicación inalámbrica para transmitir los datos utilizando una red local o remota, hacia un servidor central para almacenar toda la información captada por cada uno de los módulos, para su posterior análisis y registro.

En este subsistema se mide el flujo y la presión de aire que proviene de la BVM. El diseño inicial contemplaba la medición utilizando un tubo de Venturi fabricado en una impresora 3D con material PLA, por el cual pasa el Vt que se entrega al paciente. Para las velocidades de aire y dimensiones del tubo de Venturi utilizados, la probabilidad de un flujo turbulento es baja, permitiendo realizar mediciones con bajos porcentajes de pérdidas (Jitschin, 2004). Sin embargo, con este diseño no era posible obtener la resolución mínima de flujo requerida para el correcto funcionamiento del sistema de control.

Para dar solución al inconveniente anterior se propusieron los modelos de sensores ADP1101 y SFM3300, se realizaron mediciones de presión manométrica y flujo respectivamente. La señal de ambos sensores es muestreada por un microcontrolador Atmega328p a una frecuencia de bus $\mathrm{I}^{2} \mathrm{C}$ de $10 \mathrm{kHz}$. Para aumentar la resolución virtual del sensor de presión manométrica se realiza un proceso de decimación 
por sobre muestreo con un factor de 25 para incrementar en dos el número de bits a cambio de una reducción de la frecuencia de muestreo a $400 \mathrm{~Hz}$ (Carrato et al., 2020). Los datos calculados y medidos son empaquetados y enviados utilizando el protocolo $\mathrm{I}^{2} \mathrm{C}$.

Para las mediciones de presión se obtuvo una resolución efectiva de 12.32 bits en la adquisición de datos del sensor (Figura 2) y el sensor de flujo tiene una resolución de 14 bits determinada por el fabricante. Se obtuvo una velocidad máxima de mediciones de 2.5 ms. Las cuales tienen las siguientes características:

- Presión manométrica hasta 4,042.25 Pa con una resolución de $0.8 \mathrm{~Pa}$.

- Flujo de hasta $250 \mathrm{~L} / \mathrm{min}$ con una resolución de $3.4 \mathrm{dl} / \mathrm{min}$.

\section{Sistema de control}

Es la parte central del sistema encargada de tomar decisiones y recibir señales de los otros dos microcontroladores. Maneja los controladores de los motores en la parte mecánica y transmisión de datos a la interfaz gráfica de usuario (GUI). El diseño está basado en un microcontrolador ATmega328p.

El flujo es controlado con una acción de control proporcional-integral-derivativa (PID) para controlar indirectamente el volumen; sin embargo, también se limita la presión máxima, se modulan las respiraciones por minuto, la relación de I:E y el volumen máximo de aire inspirado. El control PID es capaz de modular el comportamiento de los motores, regulando la velocidad y torque de los mismos. La presión máxima es controlada directamente por los datos obtenidos del sensor, por lo que, si se detecta que se está excediendo el umbral, la velocidad de los actuadores es corregida para disminuir el exceso de presión en los pulmones del paciente para evitar barotrauma.

Las respiraciones por minuto (rpm) del paciente, se describen en unidades $\min ^{-1}$ y la relación I:E se representa como fracción ie $=\mathrm{I} / \mathrm{E}$. El volumen máximo se estima a través de los datos obtenidos del sensor de flujo, al integrarlo en el tiempo de operación, durante el proceso de inspiración.

Con base en esta señal periódica, se solicitan los datos a los sensores y se actualizan los nuevos valores de los actuadores.

Interfaz de motores. Este módulo es el encargado de tomar las instrucciones del módulo de control (incluido en el mismo microcontrolador), convertirlas en códigos de pasos y transmitirla a los controladores que manejan los motores paso a paso (Stepper) NEMA 17 (CUI Devices, 2020). Se utilizan circuitos integrados A4988 (Allegro MicroSystems, 2014) para controlar los actuadores y poder suministrar la potencia que estos requieren para el funcionamiento del respirador. Este control integra un inicio y un fin de carrera, el cual le indica al sistema donde inicia el recorrido cuando esté activo, lo que significa que la BVM está totalmente libre, esto se emplea en cada ciclo de respiración. El final de carrera colocado es de manera preventiva, a modo de que cuando algún sensor falle y el sistema siga informando al motor que compresione la BVM, este tenga un punto en el que ya no pueda avanzar más.

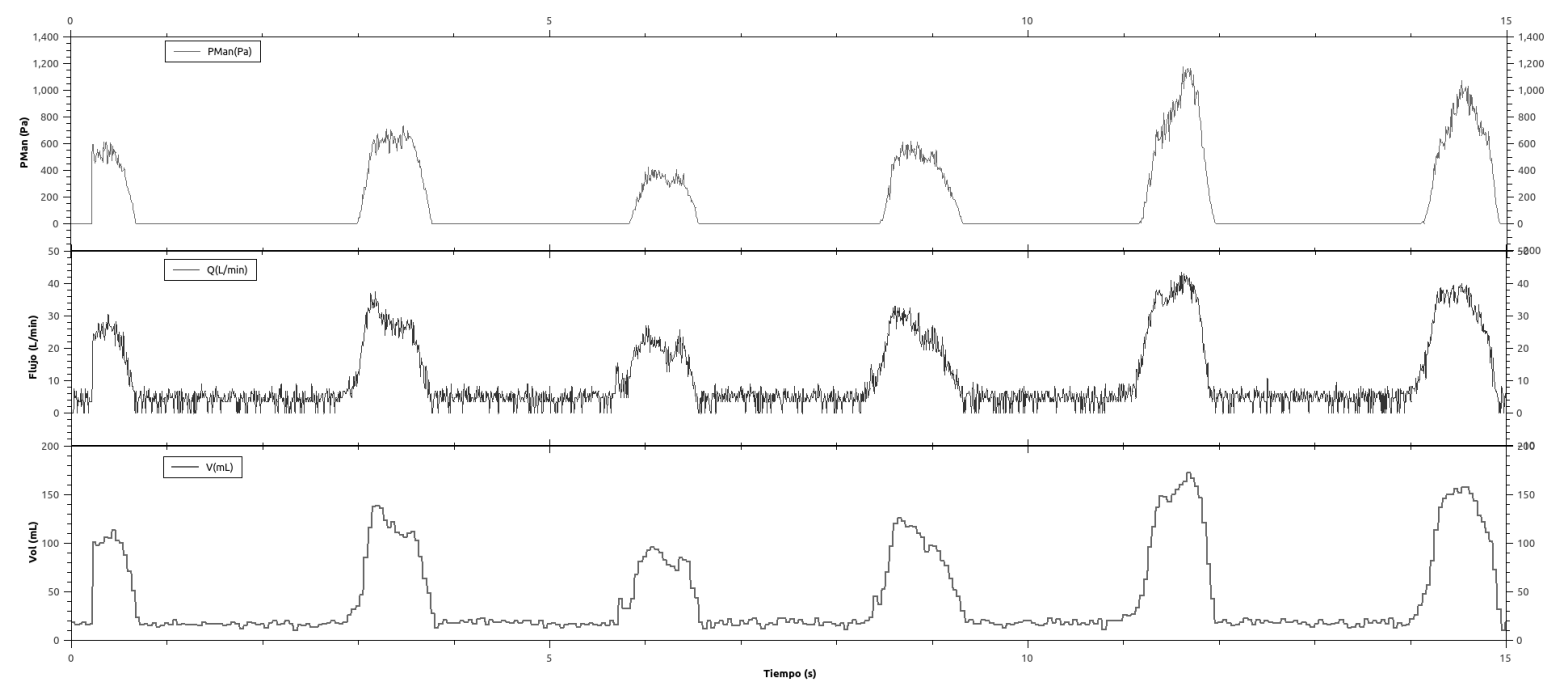

Figura 2. Gráficos obtenidos a partir del sensor de presión diferencial. 
Existe un sistema de seguridad que evita que los motores presionen la BVM más allá del límite permitido, evitando un barotrauma o volutrauma en los pulmones del paciente. Esto implica que, incluso si el sensor de flujo llegase a fallar súbitamente (causando cálculos incorrectos de volumen en el sistema de control), el dispositivo mecánico se mantiene sin daños. Dicho sistema se basa en la monitorización continua de los interruptores de final de carrera y, junto a un circuito de lógica digital combinacional (externo al microcontrolador), determina si se debe detener el funcionamiento de alguno de los motores.

Tratamiento de datos. Los bloques Sensores Interfaz y GUI Interfaz son los encargados de la comunicación con los otros dos microcontroladores, el primero se encarga de recibir los datos bajo pedido (configuración maestro) mediante un protocolo de comunicación serial $\mathrm{I}^{2} \mathrm{C}$. Este a su vez ingresa los datos recibidos en una memoria FIFO (del inglés First In First Out), donde estarán disponibles para ser procesados por la unidad de control y el módulo de interfaz gráfica. Por último, el bloque GUI Interfaz, es el encargado de enviar información bajo el pedido del microcontrolador de interfaz de usuario bajo una configuración esclavo; así mismo, es donde se notifica al usuario final si uno de los sensores está recibiendo lecturas anormales, por ejemplo, si el nivel de estrés es muy alto, el sensor toma esta información y el módulo activa una alarma para que se rectifique alguna operación y alertar al médico.

\section{Control electrónico del dispositivo}

El dispositivo cuenta con un sistema de control electrónico de lazo cerrado que se encarga de realizar ajustes en tiempo real, para que los parámetros de ventilación determinados por los médicos se mantengan invariables. Esto se logra con el uso de una estrategia de control basada en cinco módulos, que ha sido implementada dentro de un microcontrolador ATmega328P independiente de los sistemas de toma directa de datos, de manejo de interfaz e ingreso de parámetros médicos (Figura 4).

Módulo de sensores. Recibe todos los datos enviados por el microcontrolador que maneja los sensores colocados en el dispositivo. Todos estos datos se recibirán por medio del protocolo de comunicación serial $\mathrm{I}^{2} \mathrm{C}$. La configuración del microcontrolador que llevará los ADCs que recibirá la toma de datos directamente de los sensores está configurado como maestro y, el módulo de sensores, como esclavo. Este módulo es el encargado de almacenar la información dentro de la memoria FIFO. Tiene capacidad para tomar 100 muestras por ciclo de respiración a $35 \mathrm{rpm}$ (Figura 3).

\section{Respuesta del sensor de flujo ante estímulo}

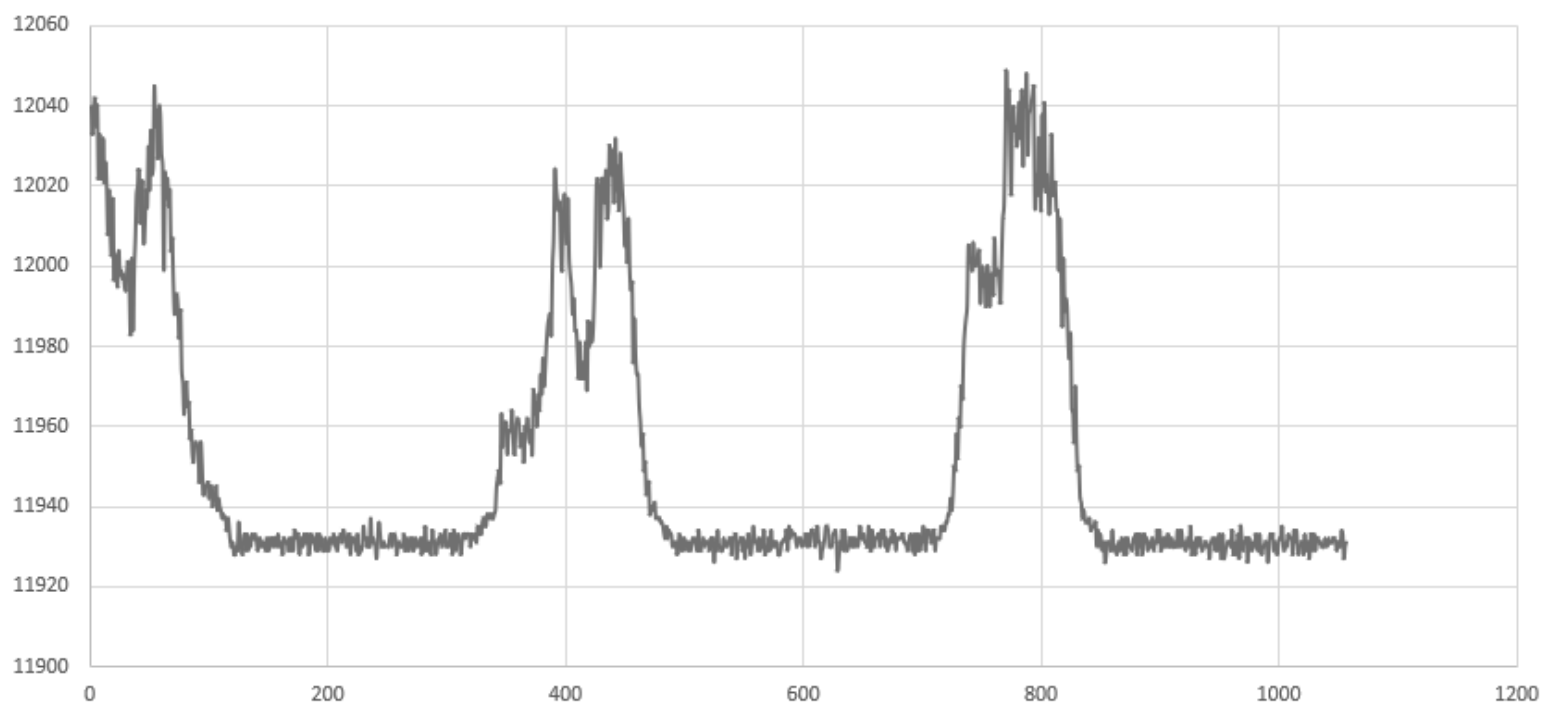

Figura 3. Resultados de sensor de flujo ante estímulo. 




Figura 4. PCB del sistema de control de AR_CODEX.

Módulo de control. Controla los motores y válvulas en función de la información almacenada en el módulo anterior. Cuenta con un sistema de control PID capaz de adaptar dinámicamente el comportamiento de la válvula y los motores, regulando la velocidad y torque de los mismos. Este módulo se encarga de establecer una vía de comunicación entre los otros dos microcontroladores encargados de la toma de datos y el control de la GUI. También controla al resto de módulos para el funcionamiento completo de todo el dispositivo, a la vez que procesa toda la información adquirida por el módulo de sensores. Toma absolutamente todas las decisiones del dispositivo electrónico por medio de la información recopilada por los sensores encargados de leer las variables y sus niveles de interés. Dentro de este módulo se realizan los cálculos de flujo y volumen total. Las variables que se controlan son: relación I:E, frecuencia ventilatoria, volumen corriente y el límite de presión.

Módulo de comunicación para los controladores (drivers) de los motores. Su función es tomar las instrucciones del módulo de control, convertirlas en códigos binarios y transmitirlas a los drivers que controlan los motores Stepper NEMA 17. Los drivers A4988 son utilizados para controlar los motores y suministrar la potencia que estos requieren para el funcionamiento del dispositivo. Los motores mencionados son los encargados de generar el movimiento mecánico para presionar la BVM y suministrar el Vt necesario al paciente. El Metal-Oxide Semiconductor Field-Effect
Transistor (MOSFET) de la válvula disponible tiene como objetivo que la respiración tenga una pendiente constante.

Módulo amortiguador y alarma. Guarda todos los registros binarios adquiridos por los sensores físicos, ingresa por medio de un bus de datos a una memoria FIFO, donde captura e interpreta la información. Tiene programados varios eventos encargados de alertar al módulo principal y al usuario si alguno de los sensores está recibiendo lecturas anormales, por ejemplo, si el nivel de estrés es muy alto el sensor toma esta información y el módulo activa una alarma para que se corrija alguna operación y alertar al médico.

Módulo comunicación para la GUI. Envía la información a desplegar en la interfaz gráfica para que el usuario final, en este caso el médico a cargo, pueda controlar las variables ventilatorias. Se comunica por medio del protocolo de comunicación serial $\mathrm{I}^{2} \mathrm{C}$ con otro microcontrolador específicamente programado para desplegar la interfaz gráfica. Este módulo está configurado como maestro y los controladores de los motores paso a paso, como esclavos.

\section{Sistema de monitoreo del paciente}

Se plantea un SoC (System on Chip) modelo ESP32 del fabricante Espressif Systemsb (Shanghai, Republica Popular de China), desarrollando la programación bajo la plataforma libre Arduino, al contar con 
conexión inalámbrica bajo estándar de IEEE 802.11 de doble núcleo. Las características de la unidad son las siguientes:

- CPU: microprocesador LX6 de 32 bits de doble núcleo (o núcleo único) de Xtensa, que funciona a 160 o $240 \mathrm{MHz}$ y tiene un rendimiento de hasta 600 DMIPS.

- Coprocesador de ultra baja potencia (ULP).

- Memoria: 532 kB SRAM.

- Conectividad inalámbrica: 802.11 b/g/n y Bluetooth: v4.2 BR/EDR y BLE.

- Interfaces periféricas ADC, DAC, GPIO de detección capacitiva, interfaces SPI, interfaces I2S, interfaces $\mathrm{I}^{2} \mathrm{C}$, y UART, entre otros.

- Todas las funciones de seguridad estándar IEEE 802.11 son compatibles, incluidas WFA, WPA / WPA2 y WAPI.

- Aceleración de hardware criptográfico: AES, SHA-2, RSA, criptografía de curva elíptica (ECC), generador de números aleatorios (RNG).

Pantalla TFT de $8.128 \mathrm{~cm}$ :

- Interface SPI.

- Luz de fondo blanca.

- Controlador gráfico LCD SSD1289.

- 65,535 colores con resolución de 320 x 240 .

- Touch Screen resistivo con controlador XPT2046.

- Conector para tarjetas de memoria SD.

\section{Parámetros requeridos}

- Para un paciente de peso ideal de $50 \mathrm{~kg}$, con un volumen de $6 \mathrm{~mL} / \mathrm{kg}$, se desea que la inspiración en cada respiración contenga $300 \mathrm{~mL}$ de aire.

- Si la frecuencia respiratoria es la mínima (15 rpm), se espera que el paciente reciba en total $300 \mathrm{~mL} * 15 \mathrm{rpm}=4.5 \mathrm{~L}$ de aire en $1 \mathrm{~min}(4.5$ $\mathrm{L} / \mathrm{min}$ ).

- La resolución del sensor de flujo debe ser como mínimo $2 \%$ del valor más pequeño a medir, para que al sumarse con los ruidos de cuantización, térmico y otras fuentes de distorsión, no se supere una resolución efectiva mínima del 5\%.

- La resolución requerida es entonces $(2 \%) *(4.5$ $\mathrm{L} / \mathrm{min})$. Las unidades de flujo del sensor están en $\mathrm{dL} / \mathrm{min}$, por lo que la resolución mínima es $(2 \%)$ * ( $45 \mathrm{dL} / \mathrm{min}$ ), lo que corresponde a $0.9 \mathrm{dL} / \mathrm{min}$.

- Además, el sensor envía una señal que no se encuentra nivelada en $0 \mathrm{~V}$. Por lo cual se procesan los datos digitales utilizando los algoritmos proporcionados por el fabricante en el microcontrolador para obtener las señales del paciente.

Se efectuaron pruebas continuas en periodos de $48 \mathrm{~h}$ con el objetivo de validar el buen funcionamiento y operación por cada grado de libertad. Las evaluaciones del sistema mecánico se realizaron simulando una carga equivalente a un pulmón de adulto, en cada una de las evaluaciones se logró comprobar la correcta operación de los motores. Se realizaron seis pruebas mecánicas con la simulación de un pulmón artificial adulto y en las seis se obtuvo resultados positivos del sistema mecánico de AR_CODEX.

Se efectuaron mediciones de temperatura en los motores en dos escenarios; se determinó que al trabajar con un solo motor se alcanzan temperaturas de $70^{\circ} \mathrm{C}$ funcionando a una frecuencia de $15 \mathrm{rpm}$. Al operar con dos motores la temperatura medida fue de $45^{\circ}$ a una frecuencia de $30 \mathrm{rpm}$. Se determinó que con la implementación de un segundo motor se obtiene el doble rendimiento del sistema mecánico y se alarga el tiempo de vida de los motores.

Basados en el sistema de control desarrollado en el dispositivo, este ha sido capaz de procesar en tiempo real hasta 250 operaciones/seg, se realizaron varias pruebas en tiempo real. El método de control de volumen se realiza indirectamente, por medio de la integración temporal del flujo. Esto permite realizar las mediciones colocando un sensor en la línea de aire que sale de la BVM. Luego de realizar las pruebas de flujo, se determina que el sensor no es capaz de entregar los datos con esta resolución, alcanzando un mínimo de 18 $\mathrm{dL} / \mathrm{min}: 20$ veces menos de lo requerido. Se requiere más que un filtro digital FIR y un filtro digital IIR paso-bajo para aumentar la resolución de la señal. Se determina que la frecuencia de operación y memoria dinámica del microcontrolador no bastan para entregar resultados aceptables.

Para las mediciones de presión, flujo y volumen se obtuvo una resolución efectiva de 12.32 bits en la adquisición de datos de los sensores (Figura 2). Se obtuvo una velocidad máxima de $8 \mathrm{~ms}$. Las cuales tienen las siguientes características:

- Presión manométrica hasta 4,042.25 Pa con una resolución de $0.8 \mathrm{~Pa}$.

- Flujo de hasta $245 \mathrm{~L} / \mathrm{min}$ con una resolución de $3.3 \mathrm{~L} / \mathrm{min}$.

- Volumen de hasta $102 \mathrm{~mL}$ con resolución de 1.37 $\mathrm{mL}$. 
Después del cambio de sensor de flujo a un dispositivo comercial de grado médico (Sensirion SFM3300), se realizó la calibración de los parámetros PID del sistema de control. Se realizaron pruebas de flujo y límite de motor utilizando el prototipo de un motor. La verificación de mayor tiempo mantuvo un comportamiento predecible y constante durante tres horas continuas. Luego, se repitió el mismo procedimiento utilizando el otro controlador de motor (dentro del mismo sistema) para verificar que el funcionamiento fuese simétrico en ambos actuadores. Los parámetros de funcionamiento de las pruebas variaron en un volumen de inspiración entre 250 y $350 \mathrm{~mL}$, I:E entre 1:2 y 1:3, así como frecuencia respiratoria en un rango de $15 \mathrm{rpm}$ a $25 \mathrm{rpm}$.

En la Figura 5, se observa una pequeña muestra del flujo medido directamente del sistema, durante la prueba de larga duración. La configuración específica utilizada en dicha fase fue un volumen de inspiración de $250 \mathrm{~mL}$, I:E 1:3 y $15 \mathrm{rpm}$.

El sistema de control fue capaz de calcular satisfactoriamente la velocidad de los motores para alcanzar la tasa respiratoria, el volumen máximo de inspiración y la relación I:E, a tal grado de regresar a la posición de inicio durante el tiempo necesario para las pruebas de frecuencias respiratorias lentas y volumen máximo bajo.

Se verificó el funcionamiento del sistema de protección de sobrepresión, que luego de modificaciones al código inicial, fue capaz de detectar una condición de peligro. Ante esta situación, el sistema de control disminuye paulatinamente la rapidez de inspiración. Se verificó que el exceso de presión no persistiera por más de $50 \mathrm{~ms}$, condición que dispara el regreso inmediato del sistema a la posición inicial, para liberar la presión y comenzar de nuevo el ciclo de inspiración. Las pruebas se realizaron con un límite de presión manométrica de $30 \mathrm{~cm} \mathrm{H}_{2} \mathrm{O}$, pero este valor puede ser modificado en la interfaz gráfica, acorde a las condiciones del paciente. Durante la prueba, se presentaron caídas aleatorias de flujo, con una periodicidad no definida, pero que luego de analizar los datos grabados de inspiración, no superan el $5 \%$ de ocurrencia.

En la Figura 6, se muestra el análisis del protocolo de comunicación entre el sistema de control y los sensores, donde se destaca la interrupción temporal en la transmisión de datos. Dicha interrupción dura en todas las ocasiones menos de $0.35 \mathrm{~s}$. Los errores se presentan al coincidir las interrupciones del protocolo, con la que genera el sistema de control del dispositivo.

En la Figura 7, aparece la interrupción temporal del flujo de aire durante la operación del sistema.
Nótese el aumento de la rapidez del flujo luego de la interrupción: esta es la compensación que se realiza automáticamente para mantener el volumen de inspiración y la tasa respiratoria en el rango de operación esperado.

También se verificó el funcionamiento adecuado de los circuitos de protección de motores y de la BVM, los cuales detienen el funcionamiento del sistema (temporalmente), en el caso que alguno de los motores sobrepase los límites de presión sobre la BVM. Dicho mecanismo es independiente del microcontrolador, por lo que, incluso ante un fallo inminente del sistema de control central, la mecánica del sistema se preservará intacta.

Finalmente, se hicieron pruebas de interacción con el sistema de interfaz gráfica. Los resultados demostraron que, incluso durante la operación continua, el operador del sistema puede ingresar nuevos parámetros de respiración para el paciente (en tiempo real), y luego de una pequeña pausa (correspondiente a un ciclo de respiración), el sistema de control actuará con base a los nuevos parámetros ingresados.

\section{Discusión}

La comparación de AR_CODEX con otros dispositivos similares no es posible, ya que por un lado existen pocos dispositivos de asistencia ventilatoria basados en BMV que utilicen un sistema de lazo cerrado, y por otro lado, los pocos que existen aún están en fase de prototipo y no se cuentan con los datos necesarios para su comparación. No es factible comparar dispositivos de lazo abierto con dispositivos de lazo cerrado, ya que una base fundamental de los dispositivos que pueden mantener mediciones estables es la adquisición de datos y el sistema de control que trabaja con esos datos. En un sistema de control de lazo abierto, simplemente no se tiene un retorno de adquisición de datos, y en la gran mayoría, no se tienen datos. De igual manera los dispositivos que han salido de lazo cerrado de este tipo, aun no han sido probados en humanos. Es importante recalcar que este dispositivo tampoco ha sido probado en animales ni humanos, algo que deberá realizarse en el futuro.

Cuando los motores NEMA-17 trabajan paralelamente, recorren la mitad de la trayectoria, por lo que se mantiene la misma velocidad y se duplica la frecuencia respiratoria máxima sin necesidad de generar más estrés a los motores. Esto significa que el dispositivo puede funcionar con ambos motores, pero si ocurre un 


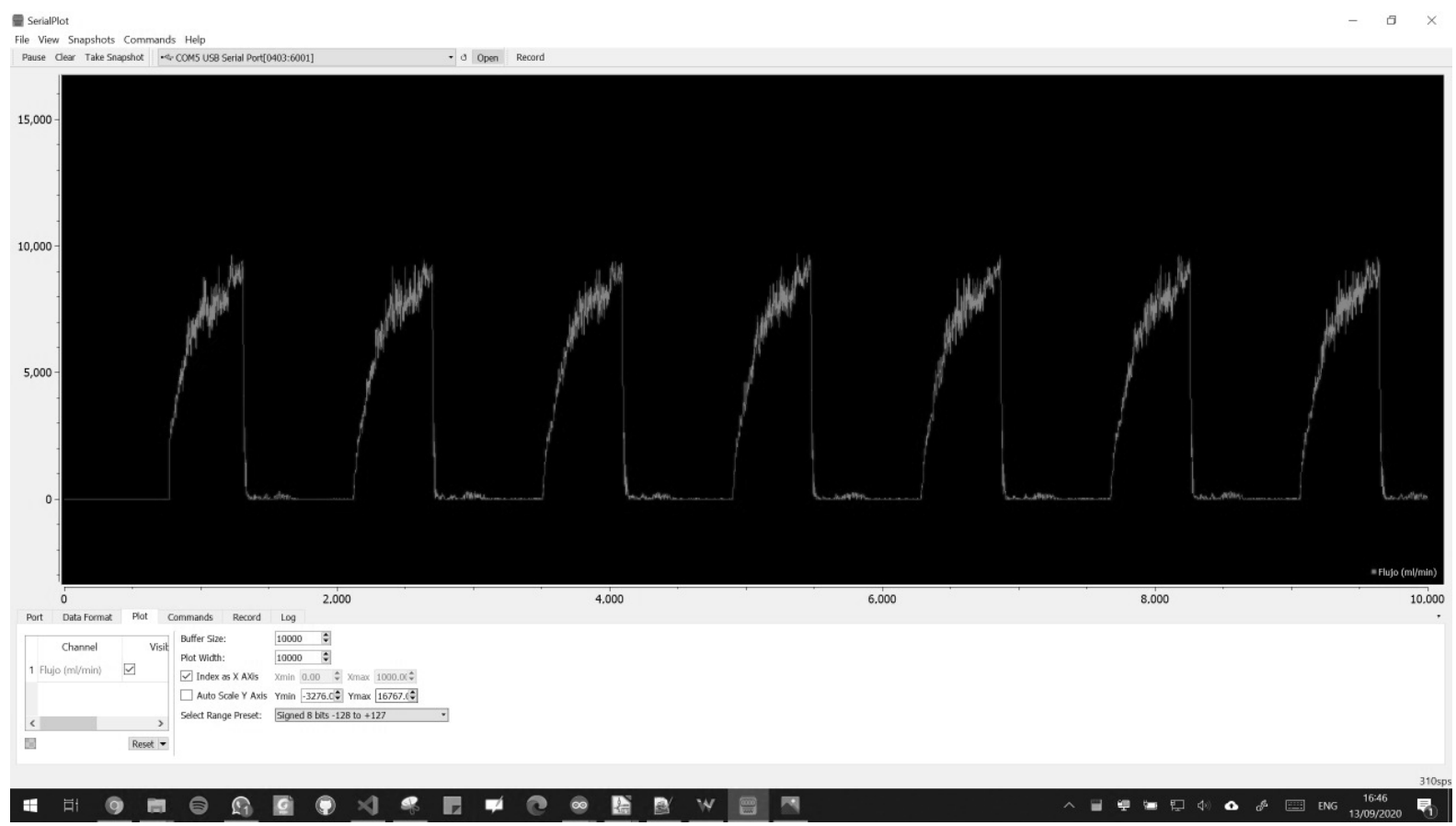

Figura 5. Flujo de aire durante la prueba de larga duración.

W Wwereforms (new workspace)

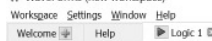

(2)

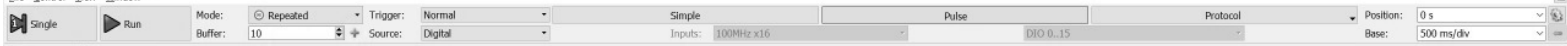

$+\cdots, \nabla \cdot$



sa.

Figura 6. Comunicación $\mathrm{I}^{2} \mathrm{C}$ entre sensores y sistema de control.

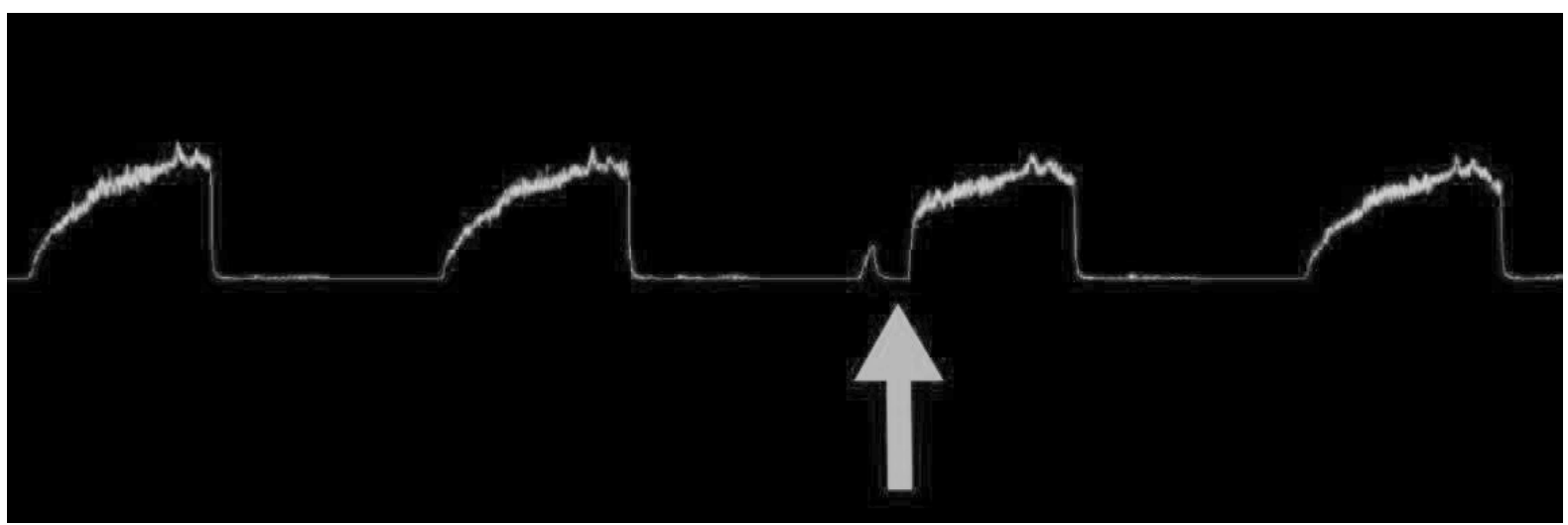

Figura 7. Interrupción de flujo compensada por el sistema de control. 
daño mecánico en algún motor, el dispositivo puede continuar trabajando con un motor. Además, los motores demostraron no superar los $70^{\circ} \mathrm{C}$ con los parámetros de operación que se definieron, esto es importante ya que asegura la integridad de los componentes fabricados en PLA al no alcanzar temperaturas que puedan ocasionar su fundición.

En el desarrollo electrónico de sensores, los datos obtenidos por el ADC se les aplicó un factor de decimación de 25. Luego de la cuantificación, se amplió la resolución efectiva de 10 bits a una resolución de 12.32 bits, aumentando la sensibilidad de las mediciones del sensor de presión manométrica y sensor de presión diferencial. El cálculo de la presión manométrica se obtuvo directamente de la curva característica del sensor, mientras que el cálculo del flujo se realizó por medio del principio de Bernoulli a través del tubo Venturi y el cálculo del volumen por medio de integración en el tiempo del flujo.

El rango de presión manométrica se mantiene dentro de los valores específicos para el uso médico, con una resolución constante, en un rango de medición entre 0.8 y $4.042 \mathrm{~Pa}$. Por otro lado, la medición del flujo de aire no es la adecuada ni la ideal para un sistema de control, debido a que la resolución obtenida no es capaz de cuantificar flujos más rápidos necesarios para el sistema de control; sin embargo, entra en los rangos con especificaciones médicas. El volumen al ser integrado en el tiempo, obtiene una resolución con mayor precisión y en una escala dentro del uso médico. Se requiere que el sensor de flujo sea implementado nuevamente, utilizando una alternativa capaz de proveer mayor resolución, sin dejar por aparte la tasa a la que se entregan los datos $(250 \mathrm{~Hz})$.

En este caso, utilizar mayor decimación del lado de sensores no resolvería el problema, ya que implicaría disminuir la frecuencia de muestreo efectiva de la señal, implicando una disminución significativa en la velocidad del sistema completo. Debido a la naturaleza del sistema, controlando el volumen inspirado (flujo neto en cada ciclo) y limitado por presión, la resolución requerida por el sensor de flujo debe entregar una resolución 20 veces mayor a la obtenida.

El cambio al sensor de flujo dedicado Sensirion SFM3300 ha permitido obtener las muestras de datos en tiempo real con resolución mayor a la requerida, por lo que el sistema de control es ahora alimentado con la información de retroalimentación necesaria para un rendimiento óptimo.

Las caídas de flujo aleatorias que se presentaron probablemente se debieron a las interrupciones que se generan en la comunicación con la placa de sensores, lo cual es un problema del microcontrolador utilizado para el sistema de control. A pesar de ello, el volumen entregado y el tiempo se mantuvieron en los rangos aceptables durante todo el período de prueba. $\mathrm{La}$ arquitectura de control diseñada garantiza estabilidad de entrega incluso con las interrupciones de la señal mencionadas, lo que resalta la importancia de un controlador de lazo cerrado en esta aplicación (calibrado adecuadamente), ya que demuestra compensar variaciones aleatorias en el flujo de aire causadas por variables externas.

Los sistemas implementados para la protección del paciente y el dispositivo, han demostrado la capacidad de actuar en menos de un ciclo de respiración y, en conjunto con la estrategia de control de lazo cerrado, se complementan para asegurar el funcionamiento adecuado bajo las condiciones a las que fueron sometidas durante la prueba del dispositivo.

\section{Agradecimientos}

Los autores agradecen a la Dirección General de Investigación (DIGI) por la ayuda económica dirigida al proyecto por medio de cheque No. 167097 de la cuenta de gastos de funcionamiento 3-033-34532-6.

$\mathrm{Al}$ equipo que desarrolló AR_CODEX, conformado por voluntarios, tanto estudiantes como egresados de la Universidad de San Carlos, de diferentes disciplinas, quienes brindaron su tiempo y trabajo para la realización del proyecto.

\section{Referencias}

Allegro MicroSystems. (2014). DMOS Microstepping driver with translator and overcurrent protection. https://www.pololu.com/file/0J450/a4988\%20 DMOS $\% 20$ microstepping $\% 20$ driver $\% 20$ with\%20translator.pdf

Australian and New Zealand Intensive Care Society. (15 April, 2020). COVID-19 Guidelines Version 2. https://www.anzics.com.au/wp-content/ uploads/2020/04/ANZI_3367_Guidelines_V2.pdf

Berlin, D. A., Gulick, R. M., \& Martinez, F. J. (2020). Severe Covid-19. New England Journal of Medicine. https://doi.org/10.1056/ NEJMcp2009575 
Carrato, S., Chatterjee, C., Cicuttin, A., Ciliberti, P., Crespo, M., Torre, S., ... Zhao, Y. (2020). A scalable High Voltage Power Supply System with system on chip control for micro pattern gaseous detectors. Nuclear Instruments and Methods in Physics Research, 963, Article 163763. https:// doi.org/10. 1016/j. nima. 2020. 163763

Collaboration, A. (2020). https://ar-codexgt.blogspot. com/

CUI Devices. (February 20, 2020). Series: Nema17. https://www.cuidevices.com/product/resource/ nema17-amt112s.pdf

Cunningham, A. C., Goh, H. P., \& Koh, D. (2020). Treatment of COVID19: Old tricks for new challenges. Critical Care, 24(91). https://doi. org/10.1186/s13054-020-2818-60

Gutiérrez, F. (2011). Ventilación mecánica. Acta Médica Peruana, 28(2), 87-104.

Iyengar, K., Bahl, S., Vaishya, R., \& Vaish, A. (2020). Challenges and solutions in meeting up the urgent requirement of ventilators for COVID-19 patients. Diabetes \& Metabolic Syndrome: Clinical Research \& Reviews, 14(4), 499-501. https://doi.org/ 10.1016/j.dsx.2020.04.048

Jitschin, W. (2004). Gas flow measurement by the thin orifice and the classical Venturi tube. Vacuum, 76(1), 89-100. https://doi.org/10.1016/j. vacuum.2004.05.014

Li, H., Li, E., Krishnamurthy, D., Kolbay, P., Chacin, B., Hoehne, S., ... Prakash, M. (2020). UtahStandford Ventilator (Vent4US): Developing a rapidly scalable ventilator for COVID-19 patients with ARDS. MedRxiv. https:// doiorg/10.1101/2020.04.18.20070367

Li, L., Li, R., Wu, Z., Yang, X., Zhao, M., Liu, J., \& Chen, D. (2020). Therapeutic strategies for critically ill patients with COVID-19. Annals of Intensive Care, 10(1). https://doi.org/10.1186/ s13613-020-00661-z
Li, X., \& Ma, X. (2020). Acute respiratory failure in COVID-19: Is it a 'typical' ARDS? Critical Care, 24(198). https://doiorg/10.1186/s13054020-02911-9

Monolithic Power Systems. (2020). MPS Open-Source Ventilator. https://www.monolithicpower.com/ en/mps-open-source-ventilator

Pawar, R. P., Tekale, S. U., Shisodia, S. U., Totre, J. T., \& Domb, A. J. (2014). Biomedical applications of poly(lactic acid). Recent Patents on Regenerative Medicine, 4(1), 40-51. https://doi.org/10.2174/221 0296504666140402235024

Pearce, J. M. (2020). A review of open source ventilators for COVID-19 and future pandemics [version 2; peer review: 3 approved]. F1000Research, 9. https://doi.org/10.12688/f1000research.22942.2

Preckel, B., Schultz, M. J., Vlaar, A. P., Hulst, A, H. Hermanides, J. de Jong, M. D., \& Hollmann, M. W. (2020). Update for anaesthetists on clinical features of COVID-19 patients and relevant management. Journal of Clinical Medicine, 9(5), 1495. https://doi.org/10.3390/jcm9051495

Whittle, J. S., Pavlov, L Sacchetti, A. D., Atwood, C., \& Rosenberg, M. S. (2020). Respiratory support for adult patients with COVID-19. Journal of the American College of Emergency Physicians Open, 1(2), 95-101. https://doi.org/10.1002/ emp2.12071

Whitwam, R. (March 26, 2020). MIT develops cheap, open source ventilator for coronavirus treatment. https://www.extremetech.com/extreme/308236mit-develops-cheap-open-source-ventilator-forcoronavirus-treatment

World Health Organization. (2020). WHO Coronavirus Disease (COVID-19) Dashboard. Recuperado el 24 de septiembre de 2020, de https://covid19.who. int 